\title{
Guest editorial: special issue on the emerging applications of neural networks
}

\author{
Tommy W. S. Chow $\cdot$ John Sum
}

Received: 26 May 2010/Accepted: 14 July 2010/Published online: 23 July 2010

(c) Springer-Verlag London Limited 2010

It has been over 60 years since the first neural network model was developed by McCulloch and Pitts [1], and has been just over 50 years since the Mark I Peceptron model was developed by Rosenblatt and Wightman [2]. The downfall of Neural Network research was due to the limited capabilities of perceptron which was rigorously proved in the book "Perceptrons" by Minsky and Papert [3]. Neural network research has then gone through a period of quiet years from the mid sixties to the mid eighties. The take off of neural computing research was probably due to the announcement that Japan had resumed the research of neural networks. The US feared that they would be overtaken by the Japanese. This resulted in Ira Skurnick, a program funding manager in the US defense Science Office, decided to fund neural computing research again. To all neural computing researchers, the late eighties are well remembered because we saw how neural computing had been reinstated and repositioned. In 1986, we saw the first annual Neural Networks for Computing Conference, and we saw Rumelhart reported back his famous backpropagation learning algorithm. To date, we have seen the flourish of neural computing with its application stretched from rigorous mathematical proof to different engineering, physical science, and even business finance applications.

This special issue on "The Emerging Applications of Neural Networks" is aimed at disseminating the latest applications of neural networks in the areas of engineering or computer science, business and management, geology

T. W. S. Chow

City University of Hong Kong, Kowloon, Hong Kong

J. Sum $(\bowtie)$

National Chung Hsing University, Taichung, Taiwan, ROC

e-mail: pfsum@nchu.edu.tw and library science. It is exciting to see different researchers from different countries, and cultures come up with new thinking and applications. In the twenty-first century, We may have come to a stage that science theories and basic research are more than sufficient to our daily lives. This special issue delineates how the versatile neural networks are affecting us everyday.

The papers solicited are selected from papers presented in the ICONIP 2009. Eventually, twelve papers from experts with innovation on new neural applications are invited and included in this issue. We include the paper:

1. "Dynamic obstacle identification based on global and local features for a driver assistance system" to provide readers another perspective of using neural networks for dynamic obstacle recognition.

2. "On-line learning from streaming data with delayed attributes: A comparison of classifiers and strategies" to discuss a new approach on handling on-line learning data which is an important issue for on-line machine learning research.

3. "Exchange Rate Prediction with Non-numerical Information" describing a finance application of exchange rate prediction.

4. "FMI Image Based Rock Structure Classification Using Classifier Combination" to provide a new kind of geology application.

5. "A Neural Decoding Approach to Auditory Temporal Assimilation" to introduce an interesting application on auditory temporal assimilation.

6. "Computer-based Detection and Classification of Flaws in Citrus Fruit" to describe a novel application on quality control in fruits.

7. "Decoding Ambisonic Signals to Irregular Loudspeaker Configuration based on Artificial Neural 
Network" to propose a fast algorithm to reconstruct the original sound field based on the combination of neural network and the modified Tabu search.

8. "RAST: Finding related documents based on triplet similarity" to introduce a tool for searching abstracts of scientific papers.

9. "Image Modeling with Combined Optimization Techniques for Image Semantic Annotation" to propose a combined optimization method which incorporates affinity propagation algorithm, optimizing training data algorithm, and modeling prior distribution with Gaussian mixture model to build Bayesian classifier for image semantic annotation.

10. "Automatic Image Restoration Based on Tensor Voting" to provide a tensor voting-based detection technique for image quality improvement, such as degraded images restoration and binary images enhancing.

11. "Robust Object tracking with Occlusion Handle" to introduce a novel incremental subspace based object tracking algorithm to deal with the occlusion problem.
12. "A GPU implementation for LBG and SOM Training" to describe a new application of graphics processing unit in neural network implementation.

Here, we would like to thank the authors of this special issue for accepting our invitation. Next, we would like to express our gratitude to the anonymous referees who spent time to review the articles and gave valuable comments for making the papers in better shape.

\section{References}

1. McCulloch WS, Pitts W (1943) A logical calculus of the ideas immanent in nervous activity. Bulletin Math Bio 5:115-133

2. Rosenblatt F (1958) The perceptron: A probabilistic model for information storage and organization in the brain. Psychol Rev 65:386-408

3. Minsky M, Papert S (1969) Perceptrons. MIT Press, Cambridge, MA 